\title{
Efectos del pastoreo holístico sobre la estructura y composición vegetal en praderas naturalizadas de uso ganadero, provincia de Última Esperanza, región de Magallanes, Chile
}

\author{
Effects of holistic grazing on structure and composition of naturalized prairies \\ under livestock grazing, provincia de Última Esperanza, Magellan region, Chile
}

Erwin Domínguez Díaz¹, Gabriel Esteban Oliva², John Báez Madariaga3

Ángel Suárez Navarro ${ }^{1} \&$ Claudio Pérez Castillo ${ }^{1}$

\section{Resumen}

Las praderas naturalizadas en Magallanes son agro-ecosistemas importantes pero frágiles a las variaciones climáticas y al sobrepastoreo. Desde el año 2013 se introdujo en Magallanes el Pastoreo Holístico como una nueva alternativa tecnológica de manejo intensivo, que tiene como principio, mejorar la salud del pastizal. Considerando esto, es prioritario generar información científica $y$ mantener un seguimiento permanente sobre este tipo de manejo. El objetivo de este trabajo fue analizar los efectos del Pastoreo Holístico sobre la vegetación en términos de estructura y composición. Se compararon tres estancias manejadas bajo Pastoreo Holístico (PH): La Campana, Lago Sofía y Parcela Tamara, con tres áreas bajo Pastoreo Semi-Intensivo (PSI) que incluye rotaciones con descansos cortos, en las estancias: Tres Pasos, Dos Lagunas y Parcela Colonia Isabel Riquelme, todas ecológicamente equivalentes a praderas naturalizadas que derivan de la sustitución de bosques de ñirre (Nothofagus antarctica (G. Forst.) Oerst.) ubicadas en la provincia de Última Esperanza, con un promedio de $389 \mathrm{~mm}$ lluvia anual. El estudio se realizó entre 2014-18. La hipótesis fue que el Pastoreo Holístico incrementa la cobertura vegetal, las especies palatables y la diversidad. Para ello se instalaron seis monitores de vegetación y suelos en tres campos con PH y tres de PSI siguiendo la metodología Monitores Ambientales de Regiones Aridas y Semiaridas (MARAS).

Los resultados indican que los cambios a través de los años no fueron significativos en cuanto a la cobertura ( $p>0.8450)$, pero sí para riqueza de especies $(p<0,0001)$, que fue mayor para el PSI. Además, se detectaron diferencias significativas en cobertura de especies nativas y exóticas $(p<0,0001)$, ya que estas últimas se incrementaron bajo $\mathrm{PH}$. Por otro lado, ambos manejos difirieron en cuanto a la cobertura de especies palatables y no palatables para el ganado $(p<0,0001)$, con una mayor cobertura de especies palatables en el $\mathrm{PH}$. Los cambios no fueron significativos en cuanto al índice de diversidad de Shannon ( $p>0,1710)$. Como conclusión, no se detectó un incremento de cobertura vegetal para el Pastoreo Holístico, pero hubo un reordenamiento de la dominancia de especies, al incrementar la cobertura de especies exóticas palatables, esto puede deberse a que la presión del pastoreo que disminuyó la competencia en el canopeo, favoreciendo a las especies exóticas naturalizadas como el trébol blanco (Trifolium repens) que no podían competir con las malezas invasoras como: Achillea millefolium y Hieracium prealtum. Futuros ensayos deberían considerar evaluar parámetros productivos como disponibilidad forraje, el peso y el estado sanitario animal.

\footnotetext{
Instituto de Investigaciones Agropecuarias (INIA). Casilla 277, Punta Arenas, Chile. $\$ edominguez@inia.cl

2 INTA, EEA, Santa Cruz, Argentina. Mahatma Gandhi 1322, 9400, Río Gallegos, Santa Cruz, Argentina.

3 Programa de Magister en Áreas Silvestres y Conservación de la Naturaleza Facultad de Ciencias Forestales, Universidad de Chile.
} 


\section{Palabras clave:}

Praderas naturalizadas, Palatabilidad de especies exóticas, diversidad florística, pastoreo rotativo, Patagonia.

\section{Abstract}

Naturalized grasslands in Magallanes are important but fragile agro-ecosystems that may change due to climatic variations and overgrazing. Holistic grazing (HG) has been introduced into Magallanes in 2013 as a new, management intensive technological alternative, that in principle would improve the health of grassland. Considering this, it is priority to generate scientific information and maintain a permanent follow-up on this type of management. The objective of this study was to analyze the effects on vegetation in order to assess whether holistic grazing degrades or improves the floristic structure and composition of naturalized grasslands. We compared three paddocks managed under grazing holistic (HG): La Campana, Lago Sofía and Parcela Tamara, with three equivalent areas under Semi-Intensive grazing (SIG), a traditional management in the area that includes rotations with short rests: Tres Pasos, Dos Lagos and Parcela Colonia Isabel Riquelme. All areas were ecologically equivalent and derived from substitution of ñirre forests (Nothofagus antarctica (G. Forst.) Oerst.), and located in the province of Última Esperanza, with $389 \mathrm{~mm}$ annual rainfall. The study was conducted between 2014-18, with an average rainfall of $372 \mathrm{~mm}$ which is within normal for that area. The hypothesis was that the HG would increase the vegetation cover, palatable species cover and diversity. Vegetation and soil monitors were installed in three fields with HG and three SIG following the MARAS methodology. The results indicate that changes over the years were not significant in terms of cover ( $p>0.8450)$, but significant differences were observed in species richness $(\mathrm{P}<0.0001)$, with a greater number of species for the SIG. Significant differences were also detected in cover of exotic species $(p<0.0001)$, that increased under HG and in cover of palatable species for livestock ( $p<0.0001)$, that was higher under HG. Differences were not significant in terms of Shannon's diversity index
( $p>0.1710)$. In conclusion, plant cover increase was not detected for $\mathrm{HG}$, but species dominance was rearranged, as palatable, exotic species, increased under HG. This is perhaps because grazing pressure decreased competition in the canopy, favoring naturalized exotic species such as white clover (Trifolium repens) that could not compete with invasive weeds like Achillea millefolium and Hieracium prealtum under SIG. Future trials should consider evaluating productive parameters such as forage availability, weight and animal health status.

\section{Key words:}

Naturalized grasslands, palatability of exotic species, floristic diversity, rotational grazing, Patagonia.

\section{INTRODUCCIÓN}

Las praderas naturalizadas en la provincia de Última Esperanza derivan de la sustitución de bosques principalmente de ñirre (Nothofagus antarctica (G. Forst.) Oerst.) a través de siembras de especies forrajeras, para habilitar tierras de mayor potencial productivo para el pastoreo bovino principalmente (Huber \& Markgraf, 2003). Estos agro-ecosistemas son importantes para la actividad silvo agropecuaria, pero frágiles a las variaciones climáticas y al sobrepastoreo (Pisano, 1990; Veblen et al. 1996). Desde el año 2013, algunos ganaderos introdujeron el Sistema de Pastoreo Holístico en Magallanes, como una nueva alternativa de innovación tecnológica, que se caracteriza por ser un sistema rotativo que usa períodos de pastoreo cortos e intensos combinados con largos períodos de descanso. El principio radica en bajar la selectividad de los animales sobre las plantas, reciclar nutrientes atrapados, mejorando así la salud del pastizal. Además, reduce en forma importante el uso de agroquímicos y mantiene un cuidadoso registro de las actividades (Savory \& Parson, 1980; Savory, 1991; Frank et al. 1998; Savory \& Butterfield, 1999; Savory, 2018). Considerando el creciente interés sobre el Pastoreo Holístico en la región de Magallanes y ahora en la región de Aysén, resulta prioritario generar información científica y mantener un seguimiento permanente sobre 
este tipo de pastoreo. Existen pocos ejemplos documentados de pastoreo rotativo de bovinos en Patagonia, dado que la mayor parte de la experiencia se concentra en ovinos (Anchorena et al. 2001; Borrelli, 2001). La bibliografía internacional tiene numerosos ejemplos $y$ conocidas revisiones como las de Holechek et al. (2000) y Briske et al. (2008), que encontraron poca evidencia de que el Pastoreo Holístico incremente la producción en relación al pastoreo convencional. Por otra parte, Teague et al. (2008) señalaron que en general estos ensayos se han realizado en parcelas pequeñas con esquemas experimentales rígidos donde los animales se mueven generando un "efecto manada" para romper las costras en el suelo, junto con promover el reciclado de la orina $y$ las heces (Frank et al. 1998). McCosker (2000) resumió los principios del Pastoreo Holístico como: 1. Controlar el descanso para adecuarlo a la tasa de crecimiento de las plantas, 2. Ajustar la carga animal al balance forrajero, 3. Planificar, monitorear y manejar el pastoreo, 4. Usar periodos de pastoreo cortos para incrementar la producción animal, 5. Usar una carga ganadera máxima por el tiempo mínimo posible, 6. Usar una diversidad de plantas y animales para mejorar la salud del pastizal, 7. Usar manadas lo suficientemente grandes para posibilitar el uso de pastores. El Pastoreo Holístico ha sido practicado en diferentes partes del mundo: en establecimientos de Zimbabue (Abel \& Blaikie, 1989), EEUU (Stinner et al. 1997), Sud África (Du Toit et al. 2009), Botswana, Namibia (McCosker, 2000), Australia (Massy, 2014) y Argentina (Borrelli, 2016). Un estudio en la Estancia Los Pozos, provincia de Santa Cruz, mostró que los largos periodos de descanso del $\mathrm{PH}$ indujeron el aumento de cobertura de pastos cortos palatables. Este incremento no se tradujo en un aumento de la oferta forrajera a nivel de campo, probablemente porque el crecimiento estaba limitado por una prolongada sequía (Oliva et al. 2016). Los defensores de PH presentan evidencia empírica de su potencial para mejorar simultáneamente la productividad y proteger el medio ambiente (Savory \& Butterfield, 1999; www.holisticmanagement.org). Sin embargo, el PH no se ha estudiado ampliamente desde una perspectiva científica y los resultados han sido contradictorios (Teague et al. 2008, Teague et al. 2011). Considerando estos antecedentes, la hipótesis planteada para este trabajo fue que el Pastoreo Holístico incrementa la cobertura vegetal, las especies palatables y la diversidad. Así, el objetivo de este estudio fue analizar el efecto del Pastoreo Holístico sobre la vegetación, para evaluar si degrada o mejora la estructura y composición florística de las praderas naturalizadas.

\section{MATERIALES Y MÉTODOS}

El ensayo se realizó en la comuna de Puerto Natales, provincia de Última Esperanza, entre 2014-18. Durante ese periodo, la pluviometría registrada, estuvo cercana al promedio de la provincia, con $372 \mathrm{~mm}$ de acuerdo a los registros de la estación climática de Puerto Natales (http://agromet.inia.cl). Hay que señalar que la producción de forraje del año 2017 fue particularmente abundante, producto de las precipitaciones de esa temporada. Para analizar el efecto de las actividades pecuarias sobre praderas naturalizadas, se seleccionaron dos áreas con diferentes sistemas de pastoreo: 1) Pastoreo Holístico (PH) planifica el pastoreo dando importancia a la cubierta vegetal, se caracteriza por ser un sistema del tipo rotativo intensivo, que usa períodos de pastoreo cortos e intensos combinados con largos períodos de descanso, el que ha sido implementado en las estancias: La Campana (51 ${ }^{\circ} 22^{\prime} 34.41^{\prime \prime} \mathrm{S}$., 72³4'39.28" O.), Lago Sofía (51³2'17.10" S., $72^{\circ} 31^{\prime} 39.62 ”$ O.) y Parcela Tamara (51 ${ }^{\circ} 45^{\prime} 15.25 ”$ S., 72²1'52.08” O.) y 2) Pastoreo Semi-Intensivo (PSI) este tipo de manejo corresponde a un intermedio entre el pastoreo intensivo y el extensivo con rotaciones simples que se caracteriza por un mayor apotreramiento respecto al pastoreo extensivo. De esta forma se logra una mejor utilización de la fitomasa disponible en el momento más adecuado para su consumo, el cual ha sido implementado en las estancias: Tres Pasos (51 22'42,08" S, 72³4'20,51" O), Dos Lagunas (5132'11,20" S., 72³1'23,59” O) y Parcela Colonia Isabel Riquelme (5146'12,42” S, 72²2’59,72”O). 

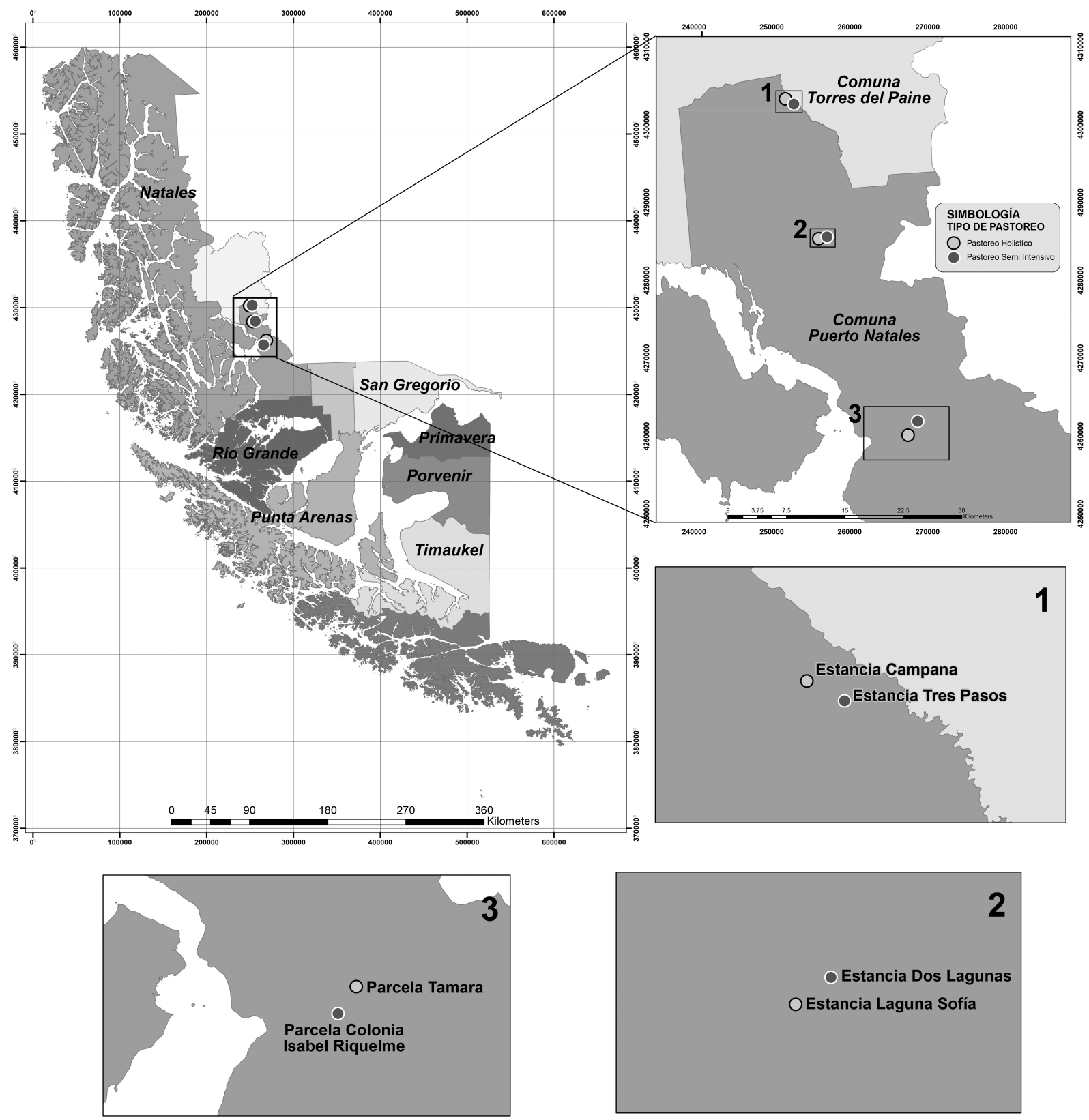

Fig. 1. Ubicación de las áreas de estudio en la provincia de Última Esperanza.

La altura de los sitios fluctuó entre los 90 y 110 m.s.n.m. (Fig. 1).

Para poder contrastar ambos tipos de pastoreo, se instalaron monitores siguiendo la metodología de Monitoreo Ambiental para Regiones Áridas y Semiáridas (MARAS), con el objeto de evaluar el efecto sobre la estructura y composición vegetal (Oliva et al. 2011, Oliva et al. 2019). La cobertura y riqueza vegetal se midió en 500 puntos a lo largo de dos transectos de $50 \mathrm{~m}$. Cada $20 \mathrm{~cm}$ se dejó caer una aguja delgada de $1 \mathrm{~m}$ perpendicular al transecto, registrando: especie vegetal, mantillo, muerto en pie, piedra y suelo desnudo. Un tercer transecto se usó para identificar parches (áreas continuas de vegetación) e interparches (áreas continuas de 
suelo desnudo que superan los $10 \mathrm{~cm}$ de largo). Posteriormente cada transecto de vegetación de $50 \mathrm{~m}$ del Monitor MARAS fue dividido en 5 subtransectos, obteniéndose un total de 10 por año ( $\mathrm{N}=150, \mathrm{PH}$ : $\mathrm{N}=150$, PSI). Luego se estimó la cobertura (expresada en porcentaje), riqueza de especies (S) y diversidad de Shannon Wiener (H') (Shannon, 1948). Con los datos obtenidos se realizó la prueba de Kruskal-Wallis con la corrección de Bonferroni, para detectar diferencias entre los factores y tratamiento, debido a que los datos no mostraron distribución normal (prueba de Shapiro-Wilk). Todos los análisis estadísticos se realizaron con INFOSTAT (Di Rienzo et al. 2016). Además, se identificaron todas las especies a nivel de género o especie según lo permitiese la muestra. La información taxonómica, relacionada al origen de las especies (nativa o exótica) fue obtenida de Fuentes (2016) y para los datos de palatabilidad se utilizaron los trabajos de Wernli (1977), Garrido \& Mann (1981), Cruz y Lara (1987) y SAG (2004).

\section{RESULTADOS}

\section{Análisis de grupos taxonómicos}

Se registraron en total 67 taxa para ambos sistemas de pastoreo, distribuidas en 55 especies, 26 familias, 49 géneros y 1 espécimen que sólo pudo ser clasificado a nivel de familia. Se presentaron en el sistema de Pastoreo Holístico 44 taxa, distribuidas en 42 especies, 24 son nativas y 20 son exóticas. En cambio, en el
Pastoreo Semi Intensivo se registraron en total 59 taxa, distribuidas en 54 especies, de ellas 40 nativas y 19 exóticas.

\section{Análisis de cobertura y riqueza de especies}

Durante cinco temporadas, se evaluó la cobertura y riqueza de especies por tipo de manejo considerando las seis unidades muestrales. Los resultados indican que no existieron diferencias significativas en cuanto a cobertura de especies entre PH y PSI, en cambio sí se establecieron diferencias significativas respecto a la riqueza de especie, siendo mayor la riqueza en el PSI (Ver Tabla 1, Figs. 2 y 3 ).

\section{Análisis del origen de las especies}

Se comparó entre PH y PSI el origen de las especies de plantas, es decir, nativas o exóticas y se relacionó el origen con la cobertura y la riqueza de especies. En la Tabla 2 y 3 se observa que existen diferencias significativas entre los manejos de acuerdo a la cobertura y riqueza de especies en relación al origen. Para el $\mathrm{PH}$ existe una mayor cobertura de especies exóticas, en cuanto a la cobertura de especies nativas no existen diferencias en cuanto al tipo de manejo (Fig. 4). Por otro parte, si existen diferencias significativas en cuanto a la riqueza de especies nativas, siendo mayor la riqueza de especies en el PSI (Tabla 3). Sin embargo, no existe significancia en cuanto a las especies exóticas entre los manejos (Fig. 5).

Tabla 1. Resultados del análisis de diferencias entre los tipos de manejo usando la prueba no paramétrica de Kruskal Wallis, para las variables cobertura y riqueza de especies. Valores de $(p>0,05)$ destacados en negrita.

\begin{tabular}{|c|c|c|c|c|c|c|c|}
\hline Variable & Tipo Manejo & $\mathrm{N}$ & Medias & D.E & Medianas & $\mathrm{H}$ & $\mathrm{p}$ \\
\hline Cobertura & $\mathrm{PH}$ & 150 & 91,54 & 8,00 & 93,82 & 2,97 & 0,0845 \\
\hline Cobertura & PSI & 150 & 90,14 & 8,49 & 92,36 & & \\
\hline Variable & Tipo Manejo & $\mathrm{N}$ & Medias & D.E & Medianas & $\mathrm{H}$ & $\mathrm{p}$ \\
\hline Riqueza & $\mathrm{PH}$ & 150 & 9,20 & 3,15 & 9,00 & 22,06 & $<0,0001$ \\
\hline Riqueza & PSI & 150 & 11,23 & 3,88 & 11,00 & & \\
\hline Tratamiento & Ranks & & & & & & \\
\hline $\mathrm{PH}$ & 126,98 & A & & & & & \\
\hline PSI & 174,02 & $\mathrm{~B}$ & & & & & \\
\hline
\end{tabular}




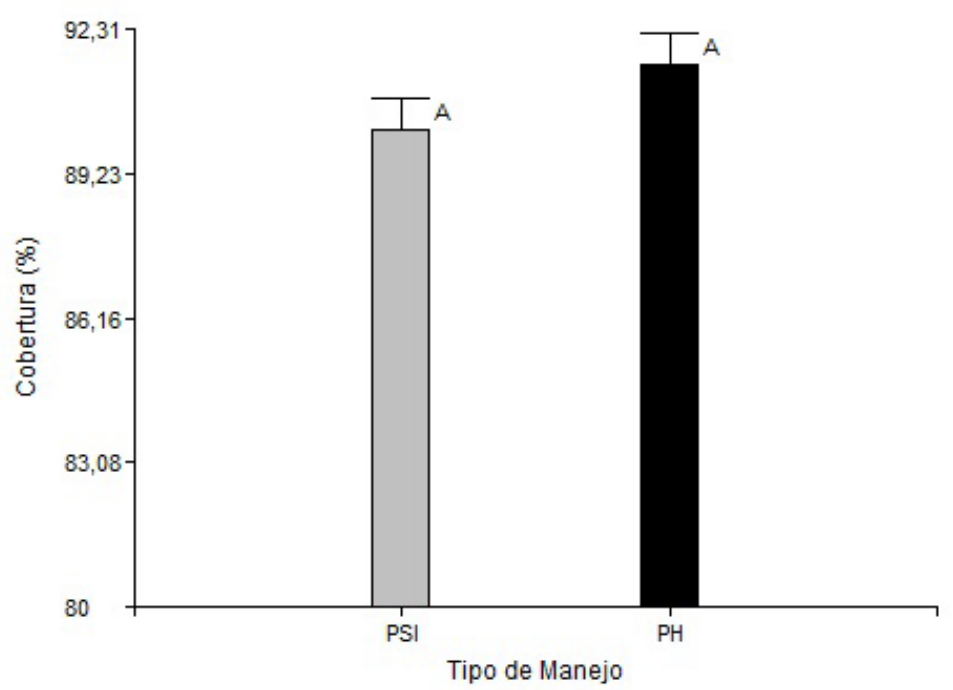

Fig. 2. Cobertura de especies (media \pm desviación estándar) según tipo de manejo. $\mathrm{PH}=$ Pastoreo Holístico y PSI = Pastoreo Semi Intensivo.

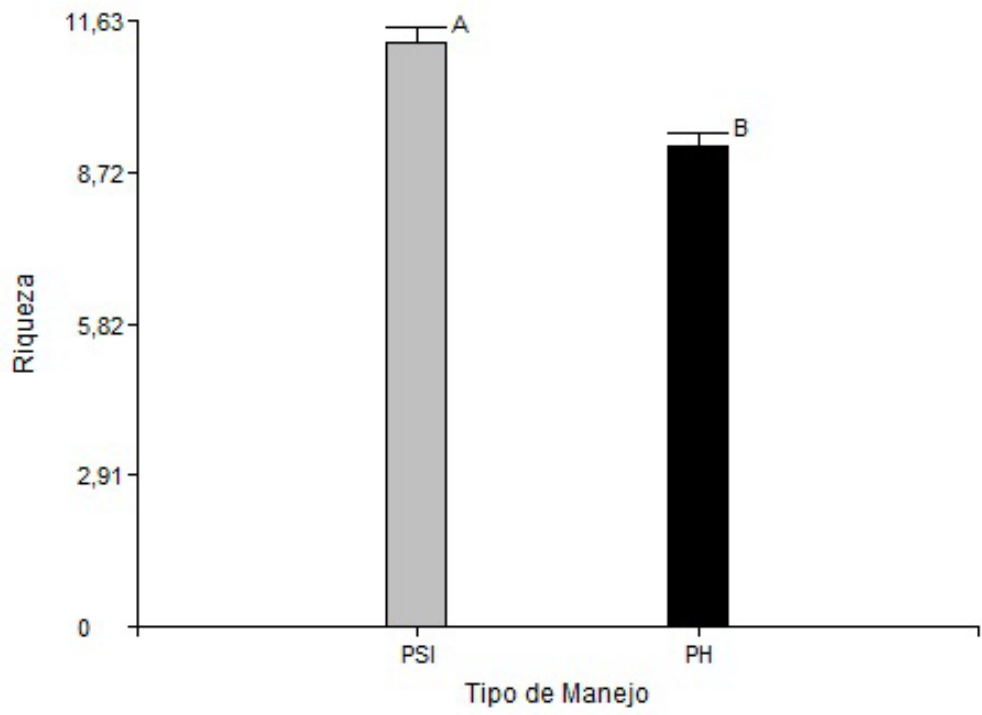

Fig. 3. Riqueza de especies (media \pm desviación estándar) según tipo de manejo. $\mathrm{PH}=$ Pastoreo Holístico y PSI=Pastoreo Semi Intensivo.

Análisis palatabilidad de las especies de plantas

Se comparó la palatabilidad de las especies en relación a la cobertura, riqueza y origen entre $\mathrm{PH}$ y PSI. En el PH existe mayor cobertura de especies palatables y además se pudo observar que no existe diferencias entre las especies palatables y no palatables entre el manejo intensivo y semi intensivo (Ver Tabla 4 y Fig. 6).

Al analizar la palatabilidad de las especies en el PSI, se determinó que existe una cantidad significativamente mayor de especies palatabes con respecto al PH. (Ver Tabla 5 y Fig. 7). 
Tabla 2. Resultados del análisis de diferencias entre los tipos de manejo usando la prueba no paramétrica de Kruskal Wallis, para las variables origen: E=Exótica y N=Nativa y su relación con la cobertura. Valores de $(p>0,05)$ destacados en negrita.

\begin{tabular}{|c|c|c|c|c|c|c|c|c|}
\hline Variable & Tipo Manejo & Origen & $\mathrm{N}$ & Medias & D.E & Medianas & $\mathrm{H}$ & $p$ \\
\hline Cobertura O & $\mathrm{PH}$ & E & 150 & 78,81 & 6,05 & 85,88 & 443,16 & $<0,0001$ \\
\hline Cobertura O & $\mathrm{PH}$ & $\mathrm{N}$ & 150 & 12,72 & 14,36 & 5,20 & & \\
\hline Cobertura O & PSI & E & 150 & 73,31 & 18,80 & 77,53 & & \\
\hline Cobertura O & PSI & $\mathrm{N}$ & 150 & 17,02 & 17,17 & 10,17 & & \\
\hline Tratamiento & Ranks & & & & & & & \\
\hline $\mathrm{PH}: \mathrm{N}$ & 136,86 & A & & & & & & \\
\hline PSI:N & 171,35 & A & & & & & & \\
\hline PSI:E & 431,85 & B & & & & & & \\
\hline PH:E & 461,94 & B & & & & & & \\
\hline
\end{tabular}

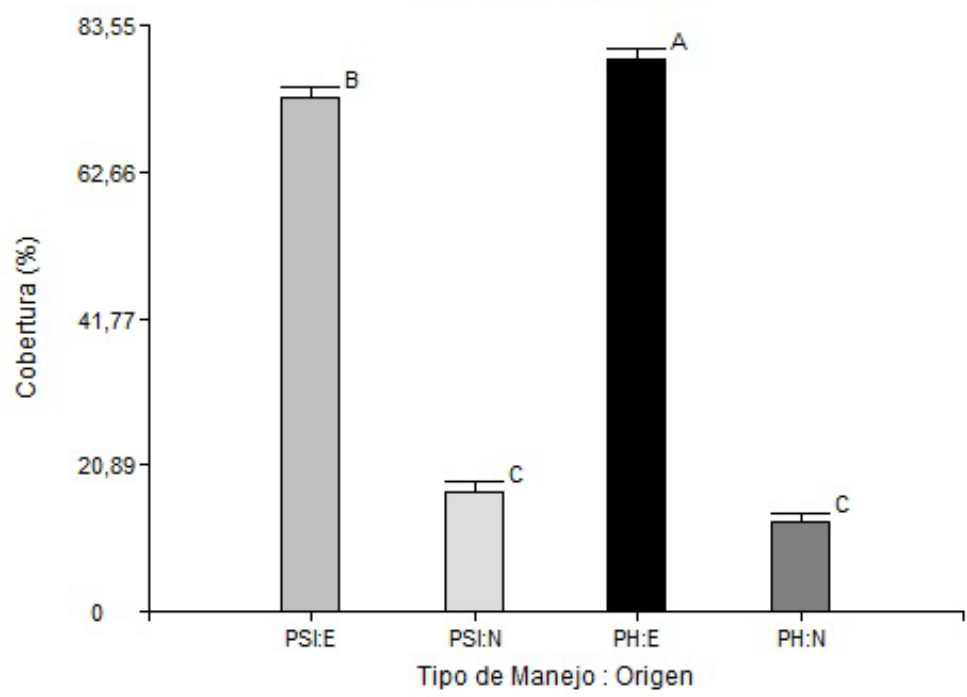

Fig. 4. Cobertura (media \pm desviación estándar) por tipos de manejos y origen de las especies. $\mathrm{PH}=$ Pastoreo Holístico y PSI=Pastoreo Semi Intensivo. E=plantas exóticas y N=Plantas nativas.

Tabla 3. Resultados del análisis de diferencias entre los tipos de manejo usando la prueba no paramétrica de Kruskal Wallis, para las variables origen: E= Exótica y N=Nativa y su relación con la riqueza de especies. Valores de $(p>0,05)$ destacados en negrita.

\begin{tabular}{|c|c|c|c|c|c|c|c|c|}
\hline Variable & Tipo Manejo & Origen & $\mathrm{N}$ & Medias & D.E & Medianas & $\mathrm{H}$ & $p$ \\
\hline Riqueza O & $\mathrm{PH}$ & E & 150 & 6,61 & 1,47 & 7,00 & 233,03 & $<0,0001$ \\
\hline Riqueza O & $\mathrm{PH}$ & $\mathrm{N}$ & 150 & 2,48 & 2,11 & 2,00 & & \\
\hline Riqueza O & PSI & E & 150 & 7,01 & 1,66 & 7,00 & & \\
\hline Riqueza O & PSI & $\mathrm{N}$ & 150 & 4,55 & 3,79 & 3,00 & & \\
\hline Tratamiento & Ranks & & & & & & & \\
\hline PH:N & 145,52 & A & & & & & & \\
\hline PSI:N & 254,66 & B & & & & & & \\
\hline PSI:E & 386,76 & C & & & & & & \\
\hline PH:E & 415,06 & $\mathrm{C}$ & & & & & & \\
\hline
\end{tabular}




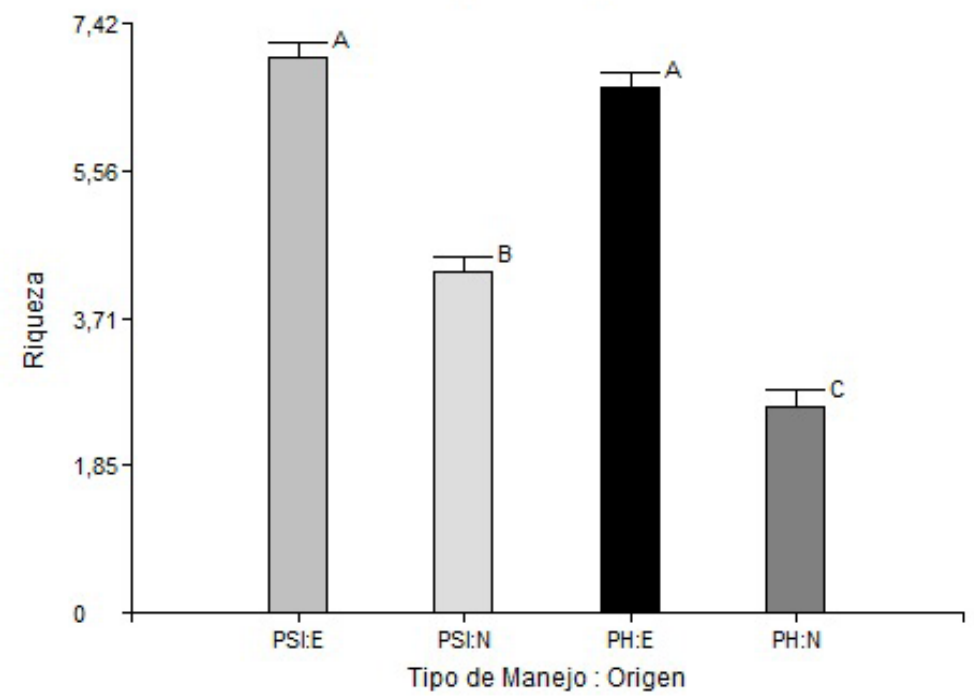

Fig. 5. Riqueza de especies (media \pm desviación estándar) según tipo de manejo y origen de las especies. $\mathrm{PH}=$ Pastoreo Holístico y PSI=Pastoreo Semi Intensivo.

$\mathrm{E}=$ plantas exóticas y $\mathrm{N}=$ Plantas nativas.

Tabla 4. Resultados del análisis de diferencias entre los tipos de manejo usando la prueba no paramétrica de Kruskal Wallis, para las variables palatabilidad: $\mathrm{NO}=$ no palatable y SI=palatable con la cobertura de especies. Valores de $(p>0,05)$ destacados en negrita.

\begin{tabular}{cccccccc}
\hline Variable & Tipo Manejo & Palatabilidad & N & Medias & D.E & Medianas & H \\
\hline Cobertura P & PH & NO & 150 & 42,62 & 9,30 & 42,30 & 54,74 \\
Cobertura P & PH & SI & 150 & 48,96 & 10,50 & 50,00 & $<0,0001$ \\
Cobertura P & PSI & NO & 150 & 48,45 & 10,76 & 48,47 & \\
Cobertura P & PSI & SI & 150 & 41,95 & 10,82 & 41,90 & \\
\hline Tratamiento & Ranks & & & & & & \\
\hline PH: SI & 245,33 & A & & & & & \\
PH: NO & 251,31 & A & & & & & \\
PSI: NO & 347,30 & B & & & & & \\
PH: SI & 358,05 & B & & & & & \\
\hline
\end{tabular}

Tabla 5. Resultados del análisis de diferencias entre los tipos de manejo usando la prueba no paramétrica de Kruskal Wallis, para las variables palatabilidad: $\mathrm{NO}=$ no palatable y $\mathrm{SI}=$ palatable con la riqueza de especies. Valores de $(p>0,05)$ destacados en negrita.

\begin{tabular}{|c|c|c|c|c|c|c|c|c|}
\hline Variable & Tipo Manejo & Palatabilidad & $\mathrm{N}$ & Medias & D.E & Medianas & $\mathrm{H}$ & $\mathrm{p}$ \\
\hline Riqueza P & $\mathrm{PH}$ & NO & 150 & 3,69 & 1,51 & 4,00 & 110,93 & $<0,0001$ \\
\hline Riqueza P & $\mathrm{PH}$ & SI & 150 & 5,54 & 2,08 & 5,00 & & \\
\hline Riqueza P & PSI & NO & 150 & 4,79 & 2,03 & 5,00 & & \\
\hline Riqueza P & PSI & SI & 150 & 6,49 & 2,73 & 6,00 & & \\
\hline Tratamiento & Ranks & & & & & & & \\
\hline PH: NO & 188,34 & A & & & & & & \\
\hline PSI: NO & 285,09 & B & & & & & & \\
\hline PH: SI & 339,08 & C & & & & & & \\
\hline PSI: SI & 389,48 & D & & & & & & \\
\hline
\end{tabular}




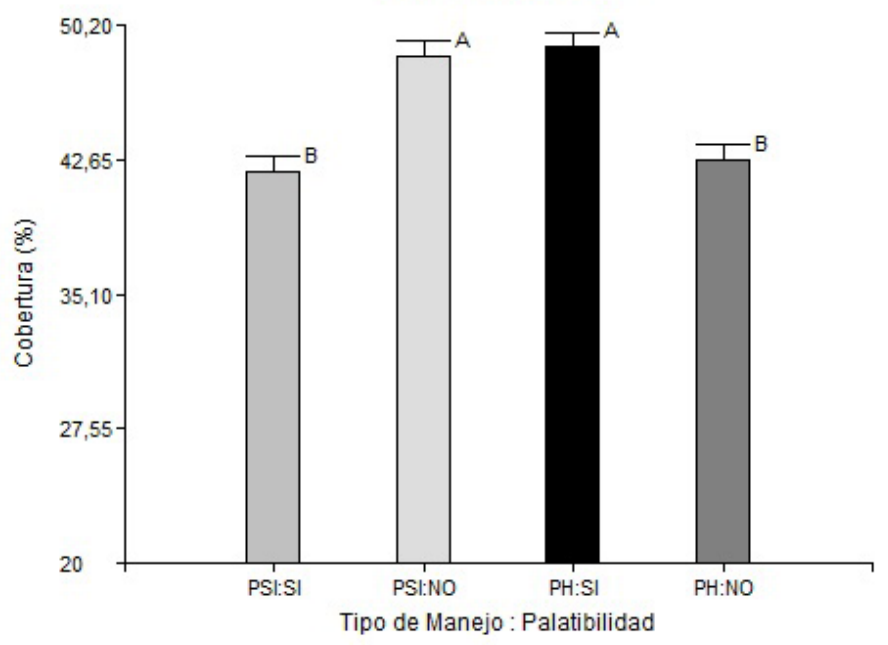

Fig. 6. Cobertura de especies palatables (media \pm desviación estándar) según tipo de manejo. PH=Pastoreo Holístico y PSI=Pastoreo Semi Intensivo. $\mathrm{NO}=$ plantas no palatables y $\mathrm{SI}=$ plantas palatables.

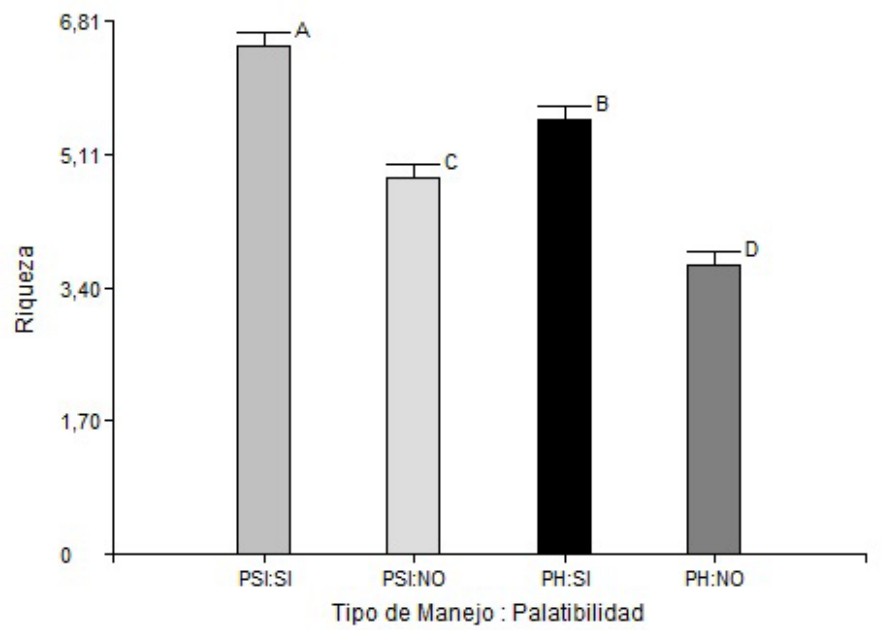

Fig. 7. Riqueza de especies palatables (media \pm desviación estándar) por tipo de manejo. $\mathrm{PH}=$ Pastoreo Holístico y PSI=Pastoreo Semi

Intensivo. $\mathrm{NO}=$ plantas no palatables y $\mathrm{SI}=$ Plantas palatables.

Tabla 6. Resultados del análisis de diferencias entre los tipos de manejo usando la prueba no paramétrica de Kruskal Wallis, para la variable diversidad.

Valores de $(p>0,05)$ destacados en negrita.

\begin{tabular}{cccccccc}
\hline Variable & Tipo Manejo & N & Medias & D.E & Medianas & H & p \\
\hline Shanon-Weaver & PH & 15 & 1,73 & 0,54 & 1,59 & \multirow{2}{*}{1,87} & 0,1710 \\
Shanon-Weaver & PSI & 15 & 1,98 & 0,51 & 1,96 & & \\
\hline
\end{tabular}


Análisis de diversidad de

Shannon-Weaver por manejo

Se observa que no existen diferencias significativas en cuanto al índice de diversidad de Shannon-Weaver (Ver Tabla 6).

\section{DISCUSIÓN}

Al comparar estadísticamente los datos de cobertura por tipo de pastoreo de cinco años de evaluación, no se detectó un incremento de cobertura vegetal, para el Pastoreo Holístico como se esperaba, de acuerdo a los estudios de Frank et al. 1998; Savory \& Butterfield, 1999; Savory, 2018. De acuerdo con algunos productores que participan del ensayo, la carga usada por el Pastoreo Holístico en los tres sitios, es aún moderada. Por otra parte, la pluviometría en los años que se realizó el estudio, mantuvo un comportamiento normal, por lo tanto, las condiciones climáticas no generaron una influencia en la selectividad de los animales al alterar la producción de plantas y la disponibilidad de ellas como ha sido descrita en otros estudios por Shewmaker et al. (1997) y Balocchi, (1999). Una variación temporal en la riqueza de especies, si pudo ser detectada claramente entre los tipos de pastoreo. Al parecer el Pastoreo Holístico genera un reordenamiento de la dominancia de las especies al incrementar la cobertura de plantas exóticas palatables versus no palatables, lo que se debería a que la presión del pastoreo disminuyó la competencia en el canopeo. Esto puede deberse a que la presión del Pastoreo Holístico a pesar de ser moderada, los animales generaron la apertura del dosel por defoliación, especialmente en aquellos lugares en donde era abundante la maleza poco palatable Achillea millefolium, que al reducir su cobertura genera un incremento en la luz, nutrientes y humedad, favoreciendo a otras especies palatables. Un comportamiento similar ha sido detectado en otros estudios al comparar el pastoreo controlado por tiempo con pastoreo continuo (Sanjari et al. 2016) $y$ en estudios en donde se compara la intensidad del pastoreo (Hickman \& Hartnett 2002). La especie exótica naturalizada más favorecida fue el trébol blanco (Trifolium repens), al inhibirse o reducirse la competencia por la luz, generada por Achillea millefolium y Hieracium prealtum. Esto permitió probablemente activar diversos puntos de crecimiento en el trébol blanco como ha sido detectado en otros cultivos en praderas (Covacevich, 2000; Griffiths et al. 2003; Romero, 2005). Similar tendencia ha sido reportada, en un estudio en la Estancia Los Pozos, de la Patagonia austral argentina, provincia de Santa Cruz, desarrollado por el INTA, el que se observó aumentos similares en la cobertura para el pastoreo continuo en comparación con el holístico, y una afectación del crecimiento de algunas de las plantas dominantes (Festuca spp.) bajo PH (Oliva col, com pers). Finalmente, en término de biodiversidad no se pudo detectar diferencias significativas, al parecer el Pastoreo Holístico en estos tres sitios es aún conservador. Se recomienda continuar con este tipo de monitoreo a largo plazo y considerar en futuros ensayos evaluar parámetros como: disponibilidad forraje, manejo animal, variaciones de peso y estado sanitario animal para comparar los resultados productivos de la implementación del manejo holístico de cada predio.

\section{AGRADECIMIENTOS}

A la Subsecretaria Ministerial de Agricultura por financiar el programa de investigación: Sistemas de Praderas Estepáricas de Zonas Frías de Chile, Código: 502093-70. Se agradece también a todos los que ayudaron en el registro de los datos en terreno: Devora Vega, Carlos Márquez, Mayte Rodríguez, Alejandra Leguen, Dazny Lemus y Paulina Santis.

\section{LITERATURA CITADA}

Abel, N. O., \& Blaikie, P. (1989). Land degradation, stocking rates and conservation policies in the communal rangelands of Botswana and Zimbabwe. Land Degradation \& Development, 1, 101-123.

Anchorena, J., Cingolani, A. M., Livraghi, E., Collantes, M.B., \& Stoffella, S. (2001). Manejo del pastoreo de ovejas en Tierra del Fuego. Buenos Aires: Edipubli S.A.

Balocchi, O. (1999). Praderas y recursos forrajeros en la zona sur de Chile. En C. Amtmann, F. Mujica \& B. Vera (Eds.), Pequeña 
agricultura en la Región de los Lagos, Chile (pp. 59-73). Valdivia: Ediciones de la Universidad Austral de Chile.

Borrelli, P. (2001). Producción animal sobre pastizales naturales. En P. Borrelli \& G. Oliva (Eds.), Ganadería ovina sustentable en la Patagonia Austral (pp. 131-162). Buenos Aires: INTA.

Borrelli, P. (2016). Regeneración de Pastizales y Cambio Climático. Buenos Aires: OVIS XXI.

Briske, D. D., Derner, J. D., Brown, J. R., Fuhlendorf, S. D., Teague, W. R., Havstad, K. M. Gillen, R. L.,... \& Willms, W. D. (2008). Rotational Grazing on Rangelands: Reconciliation of percetion and experimental evidence. Rangeland Ecology and Management 61, 3-17.

Covacevich, N. (2000). Trébol para Magallanes. Revista Tierra Adentro, 34, 40-43.

Cruz, G., \& Lara, A. (1987). Vegetación del Ârea de Uso Agropecuario de la XII Región, Magallanes y de la Antártica Chilena. Instituto de Investigaciones Agropecuarias. Estación Experimental Kampenaike. Intendencia de la XII. Región Magallanes y Antártica Chilena. Punta Arenas, Chile.

Di Rienzo, J., Casanoves, F., Balzarini, M., Gonzalez, L., Tablada, M., \& Robledo, C. (2011). InfoStat versión 2016. Grupo InfoStat, FCA, Universidad Nacional de Córdoba, Argentina. URL http://www. infostat. com. ar.

Du Toit, G. V. N., Snyman, H., \& Malan, P. (2009). Physical impact of grazing by sheep on soil parameters in the Nama Karoo subshrub/ grass rangeland of South Africa. Journal of Arid Environments, 73, 4-810.

Frank, D. A., McNaughton, S. J., \& Tracy, B. F. (1998). The ecology of the earth's grazing ecosystems. BioScience, 48, 513-521.

Fuentes, N., Pauchard, A., Sánchez, P., Esquivel, J., \& Marticorena, A. (2013). A newcomprehensive database of alien plant species in Chile based on herbarium records. Biological Invasions, 15, 847-858.

Garrido, O., \& Mann, E. (1981). Composición química, digestibilidad y valor energético de una pradera permanente de pastoreo a través del año. Tesis Licenciatura Agropecuaria. Universidad Austral de Chile, Facultad de Ciencias Agrarias. Ms.

Griffiths, W., Hodgson, J., \& Arnold, G. (2003). The influence of sward canopy structure on foraging decisions by grazing cattle. I. Patch selection. Grass and Forage Science, 58, 112-124.

Hickman, K., \& Hartnett, D. (2002). Effects of grazing intensity on growth, reproduction, and abundance of three palatable forbs in Kansas tallgrass prairie. Plant Ecology, 159, 23-33.

Holechek, J. L., Gomes, H., Molinar, F., Galt, D., \& Valdez, R. (2000). Short-duration grazing: the facts in 1999. Rangelands, 18-22.

Huber, U., \& Markgraf, V. (2003) European impact on regimes and vegetation dynamics at the steppe-forest ecotone of southern Patagonia. The Holocene, 13, 567-579.

Massy, C. (2014). A glass three-quarters full: regenerating native grassland landscapes with holistic grazing management. Grass half-full or grass half-empty? Valuing native grassy landscapes. Friends of Grasslands' forum 30 October - 1 November 2014. 1-5.

McCosker, T. (2000). Cell Grazing-the first 10 years in Australia. Tropical Grasslands, 34, 207-218.

Oliva, G. E., Bran, D., Gaitán, J., Ferrantea, D., Massara, V., Martínez, G. G., Adema, E., M. Enrique, M., Domínguez, E., \& Paredesa, P. (2019) Monitoring drylands: The MARAS system. Journal of Arid Environments 161(2), 55-63.

Oliva, G. E., Cepeda, C., Ferrante, D., \& Puig, S. (2016). Holistic management in a semiarid Patagonian sheep station: slow grassland improvement with animal production complications. In A. Iwaasa, H. A. Lardner, W. Willms, M. Schellenberg, \& K. Larson (Eds.), X The Future Management of Grazing and Wild Lands in a High-Tech World (pp. 1115-1117). Proceedings 10th International Rangeland Congress. International Rangeland Congress, Sasktatooon, Canada.

Oliva, G. E, Gaitan, J., Bran, D., Nakamatsu, V., Salomone, J., Buono, G., Escobar, 
J.,... \& Celdrán, D. (2011). Manual para la instalación y lectura de monitores MARAS. Buenos Aires: PNUD.

Pisano, E. (1990). Labilidad de los ecosistemas terrestres fuegopatagónicos. Anales Instituto Patagonia, Serie Ciencias Naturales, 19, 17-25.

Romero, O. (2005). Trébol blanco: clave en los sistemas pastoriles. Revista Tierra Adentro, Sep-Oct, 21-23.

SAG(2004). ElPastizal Última Esperanzay Navarino Guía de Uso, Condición Actual y Propuesta de Seguimiento para Determinación de Tendencia. Proyecto FNDR- SAG XII Región de Magallanes y Antártica Chilena: "Protección y Recuperación de Pastizales XII Región” BIP N²0105466-0.

Sanjari, G., Ghadiri, H., \& Yu, B. (2016). Effects of time-controlled and continuous grazing on total herbage mass and ground cover. Journal of Agriculture and Rural Development in the Tropics and Subtropics, 117(1),165-174.

Savory, A. (2013). Response to request for information on the science and methodology underpinning Holistic Management and holistic planned grazing. Savory Institute. URL http://www. savoryinstitute. com.

Savory, A. (2018). The Foundations of Holistic Management. Boulder Colorado: Savory Institute.

Savory, A., \& Butterfield, J. (1999). Holistic Management: A new framework for decision making. New York: Island Press.

Savory, A., \& Parson, S. (1980). The Savory grazing method. Rangelands, 2, 234-237.

Shewmaker, G., Mayland, H., \& Hansen, S. (1997). Cattle grazing preference among eight endophyte-free tall fescue cultivars. Agronomy Journal, 89, 695-701.

Stinner, D. H., Stinner, B. R., \& Martsolf, E. (1997). Biodiversity as an organizing principle in agroecosystem management: case studies of holistic resource management practitioners in the USA. Agriculture, ecosystems \& environment, 62, 199-213.

Teague, R., Dowhower, S. L., Baker, N., DeLaune, P. B., \& Conover, D. M. (2011). Grazing management impacts on vegetation, soil biota and soil chemical, physical and hydrological properties in tall grass prairie. Agriculture, Ecosystems and Environment, 141, 310-322.

Teague, R., Provenza, F., Norton, B., Steffens, T., Barnes, M., Kothmann, M., \& Roath, R. (2008). Benefits of multi-paddock grazing management on rangelands: limitations of experimental grazing research and knowledge gaps. Grasslands: ecology, management and restoration. Hauppauge, NY, USA: Nova Science Publishers, 41-80.

Veblen, T., Donoso C., Kitzberger, T., \& Rebertus, A. (1996). Ecology of southern Chilean and Argentinean Nothofagus forests. In T. Veblen, R. Hill, J. Read (Eds.), The Ecology and Biogeography of Nothofagus Forests (pp. 293-353). New Haven, Conneticut: Yale University Press.

Wernli, K. C., Doberti N. H., Schmitt, M. J., Alonso O., \& Cerda, D. (1977). Estudio sobre valor nutritivo de las praderas en Magallanes. Instituto de Investigaciones Agropecuarias (Chile), Estación Experimental Kampenaike (Punta Arenas). 